\title{
Exposition trotz Psychose?
}

Fragestellung: Wie wirksam und sicher sind Expositionsbehandlungen und EMDR (Eye Movement Desensitization and Reprocessing) bei Patienten mit psychotischen Erkrankungen und komorbider posttraumatischer Belastungsstörung (PTSD)?

Hintergrund: Einige Patienten mit schizophrenen oder schizoaffektiven Erkrankungen sind gleichzeitig auch an einer PTSD erkrankt. Metaanalysen zufolge liegt der Anteil dieser Komorbidität bei etwa $12 \%$ der psychotischen Patienten [1]. Für die Psychotherapie der PTSD haben sich sowohl die Methode der verlängerten Exposition (Prolonged Exposure, PE) als auch die EMDR als wirksam erwiesen. Aktuelle Leitlinien für die Therapie der PTSD empfehlen beide Therapieformen als Methoden der ersten Wahl. Therapiestudien zur PTSD schließen PTSDPatienten mit Psychosen in der Regel aus. Viele Kliniker zögern, EMDR oder PE bei psychotischen Patienten anzuwenden, weil sie eine psychotische Dekompensation unter der Psychotherapie befürchten [2]. Bislang fehlen kontrollierte Studien zur Frage, ob diese Befürchtung oder die Beurteilung der psychotischen Komorbidität als Kontraindikation gerechtfertigt ist.

Patienten und Methodik: Die einfach verblindete, randomisierte Studie schloss erwachsene Patienten zwischen 18 und 65 Jahren ein, die aktuell die Kriterien einer chronischen PTSD nach

DSM-IV-TR erfüllten und zu

Van den Berg $D$, de Bont $P$, van der Vieugel $B$ et al. Prolonged exposure vs eye movement desensitization and reprocessing vs waiting list for posttraumatic stress disorder in patients with a psychotic disorder. A randomized clinical trial. JAMA Psychiatry 2015; 72: 259-67 irgendeinem Zeitpunkt ihres Lebens an einer psychotischen oder einer affektiven Störung mit psychotischer Symptomatik erkrankt waren. Ausschlusskriterien waren unter anderem akute Suizidalität, weniger als zwei Monate zurückliegende Ände- rungen der antipsychotischen oder antidepressiven Medikation, unzureichende Sprachkenntnisse oder aktuelle Unterbringungen auf einer geschlossenen psychiatrischen Station. Insgesamt 155 Patienten schließlich wurden auf die drei Studienarmexpositionstherapie (PE), EMDR und Warteliste (WL) randomisiert. Alle Studienteilnehmer erhielten eine Behandlung der Psychose mit antipsychotischer Medikation sowie eine multidisziplinäre Unterstützung durch das Ambulanzteam. Die Patienten der EMDRund der PE-Gruppe wurden zusätzlich wöchentlich mit acht Therapiesitzungen à 90 Minuten nach der entsprechenden Methode behandelt. In der Auswertung wurden beide Therapiearme gegeneinander sowie gegen die Wartegruppe ausgewertet. Die Patienten wurden vor Studienbeginn, nach Ende der Therapiephase sowie nach sechs Monaten Follow-up untersucht. Erhoben wurden neben soziodemografischen Daten der aktuelle psychopathologische Befund und die spezifische PTSD-Symptomatik.

Ergebnisse: Von 452 untersuchten Patienten konnten nach Berücksichtigung der Ein- und Ausschlusskriterien 155 Patienten randomisiert und auf die beiden Studienarme verteilt werden (53 PE, 55 EMDR und $47 \mathrm{WL}$ ). Beide Therapiearme zeigten im Vergleich zur Wartegruppe eine signifikante Besserung der PTSD-Symptomatik nach Therapieende und zum Follow-up nach sechs Monaten. Der direkte Vergleich der beiden Therapiegruppen ergab keine signifikanten Unterschiede zwischen EMDR und PE. Psychotische Dekompensationen und ernste Nebenwirkungen wurden nicht beobachtet.

Schlussfolgerungen: Diese Studie zeigt, dass PTSD-Patienten auch bei Vorliegen einer komorbiden psychotischen Erkrankung entweder mit einer Expositionstherapie oder mit EMDR gut, wirksam und sicher behandelt werden können. Es gibt keinen Grund, solchen Patienten diese Form einer effektiven Traumatherapie vorzuenthalten.

\section{- Kommentar von Andreas Thiel und Carsten Konrad, Rotenburg \\ Wertvolle Ergebnisse für den klinischen Alltag}

Die eindeutigen Ergebnisse dieser sorgfältig randomisierten, kontrollierten und einfach verblindeten Psychotherapiestudie sprechen gegen die weitverbreitete und oft wiederholte Annahme, psychotische Erkrankungen seien grundsätzlich Kontraindikationen gegen eine Psychotherapie mit EMDR (Eye Movement Desensitization and Reprocessing) oder eine Expositionbehandlung.

Solange komorbide Erkrankungen in zahlreichen Therapiestudien Ausschlusskriterien sind, bleibt die externe Validität dieser Untersuchungen gering. Denn im klinischen Alltag begegnen uns regelmäßig Patienten mit mehreren Problemen und Diagnosen, die mit den hochausgelesenen Patienten aus Studien nur wenige Gemeinsamkeiten haben. Insofern ist diese Studie für den klinischen Alltag besonders wertvoll.
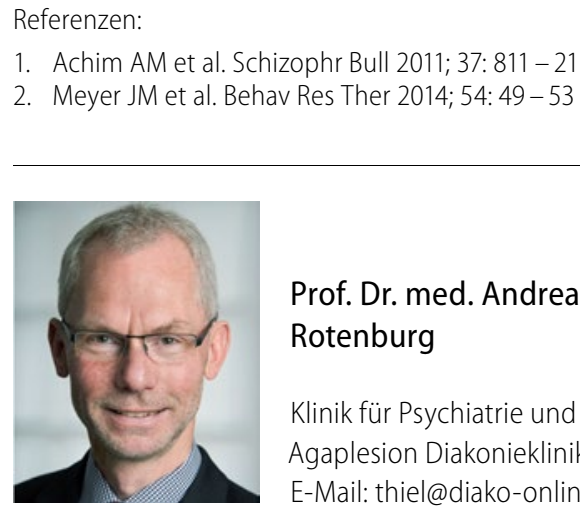

Prof. Dr. med. Andreas Thiel, Rotenburg

Klinik für Psychiatrie und Psychotherapie, Agaplesion Diakonieklinikum Rotenburg E-Mail: thiel@diako-online.de 